\title{
Poderia a Atividade Física Induzir Analgesia em Pacientes com Dor Crônica?
}

\author{
Can Exercise Induce Analgesia in Patients With Chronic Pain?
}

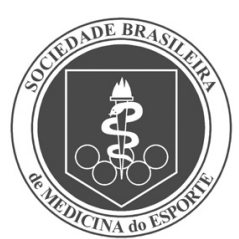

Artigo de ReVisão
Juliana Barcellos de Souza'

1. Doutora em Ciências clínicas (Sciences de la Santé) - Université de Sherbrooke Qc/Canadá

\section{Endereço para correspondência:} Rua Lauro Linhares, 2123, sala 309-A Florianópolis/SC Brasil - 88036-002 E-mail:

Juliana.Barcellos.de.Souza@gmail.com

Submetido em 22/08/2008

Versão final recebida em 04/11/2008

Aceito em 28/11/2008

\begin{abstract}
RESUMO
A dor crônica caracteriza-se pela persistência do sintoma além do período fisiológico de recuperação do tecido lesado. Essas dores causam incapacidade física e redução da performance cognitiva, reduzem a qualidade de vida e o bem-estar dos pacientes, cujo tratamento proposto contradiz o clássico binômio da terapia da dor aguda (repouso e fármacos). Para a dor crônica prescrevem-se exercícios físicos e sugerem-se tratamentos multidisciplinares. Embora a atividade física seja prescrita há mais de 20 anos, os mecanismos neurofisiológicos envolvidos ainda não são compreendidos. Descrevemos brevemente os mecanismos endógenos de controle da dor crônica e evidências da literatura científica que defendem o sistema opioide como mecanismo de ação na analgesia induzida pelo exercício em indivíduos sadios e atletas. Esse mecanismo também parece agir na população com dor crônica, embora haja controvérsias. Finalizamos o artigo com considerações clínicas para a prescrição do exercício para a população com dor crônica.
\end{abstract}

Palavras-chave: dor crônica, atividade física, mecanismos endógenos de controle da dor.

\begin{abstract}
Chronic pain is defined as persistent pain beyond normal tissue healing time. Chronic pain syndromes have a considerable impact on functional capacity, resulting in disrupted work and social activities; therefore, the impact of these syndromes affect the society at large and at a high economic cost. In contrast to rest and pharmacological treatment, multidisciplinary programs with exercises have shown to improve pain and function in chronic pain patients. A number of studies reported analgesia induced by exercise; however, the neurological mechanisms involved are not known yet. To explore this phenomenon, we describe endogenous pain control relating some studies on general population and chronic pain subjects, and we conclude this paper with some clinical consideration to determine optimal intensity of exercise to produce hypoalgesia.
\end{abstract}

Keywords: chronic pain, exercise, endogenous pain control systems.

\section{INTRODUÇÃO}

A dor é uma percepção subjetiva, desagradável e vital. A interpretação do estímulo nocivo protege o organismo através desse sinal de alarme denominado dor ${ }^{(1)}$. Num contexto temporal, a dor pode ser classificada como aguda ou crônica. A dor aguda está associada a lesão do organismo, é de curta duração e desaparece com a cicatrização dessa lesão, por exemplo dor pós-operatória. A dor crônica, por sua vez, é persistente ou recorrente e não está necessariamente associada a uma lesão no organismo. A cronificação da dor pode ser de causa desconhecida. Em sua classificação, consideram-se crônicas aquelas em que o sintoma se mantém além do tempo fisiológico de cicatrização de determinada lesão, ou por permanecer por mais de três meses ${ }^{(2-4)}$, por exemplo, as síndromes dolorosas como lombalgias crônicas ou fibromialgia.

Estima-se que, mundialmente, $80 \%$ das consultas médicas devamse à presença da dor ${ }^{(5)}$. Um recente estudo brasileiro demonstra que 75\% dos pacientes que consultam serviços públicos de saúde relatam a presença de dor crônica(6). A alta prevalência da dor crônica no Brasil torna-a um problema de saúde pública, com um impacto socioeconômico importante. Dados do INSS, em 2007, apontam que 20\% dos benefícios concedidos por afastamento do trabalho foram destinados a pacientes com dores crônicas (Dataprev 2007). Os gastos associados a essa condição de saúde estimulam o sistema a investigar intervenções eficazes para o tratamento das dores em geral ${ }^{(7)}$.

O tratamento clássico da dor (aguda) consiste em repouso e uso de fármacos para o alívio do sintoma, para favorecer a cicatrização da lesão ${ }^{(8)}$ e a redução do processo inflamatório ${ }^{(9)}$. Ao contrário do tratamento da dor aguda, os agentes químicos analgésicos não se demonstram eficazes contra a dor crônica(10,11). A baixa eficácia do uso contínuo de fármacos está inevitavelmente associada a efeitos secundários indesejáveis e à baixa adesão ao tratamento farmacológico. Aproximadamente, $47 \%$ dos pacientes com fibromialgia (síndrome de dor crônica difusa) não aderem à medicação prescrita, seja intencionalmente ou pela intensidade dos efeitos colaterais da medicação(12).

O tratamento da dor crônica caracteriza-se por programas multidimensionais agindo sobre características biopsicossociais ${ }^{(10,13)}$. No plano biológico esses programas visam regular os mecanismos endógenos de controle da dor e a concentração de neurotransmissores (como serotonina, noradrenalina e dopamina). No plano psicológico reduzem ansiedade, depressão, angústia e incapacidades mentais geradas pela dor crônica. No plano social favorecem a autoestima, a participação social e a produtividade intelectual e física. Dentre as estratégias empregadas pelos programas multidisciplinares destinados à dor crônica, temos gestão do estresse, educação dos pacientes e das famílias, psicoterapia, relaxamento, sendo a atividade física a estratégia mais utilizada ${ }^{(14,15)}$. A prescrição de exercícios para o tratamento da dor crônica é defendida há mais de 20 anos pela literatura científica ${ }^{(16)}$

Embora a atividade física seja a mais comum dentre as modalidades dos tratamentos multidisciplinares, o efeito analgésico do exercício ainda é contraditório. Enquanto alguns estudos clínicos demonstram a redução da dor após a atividade física(17), outros relatam a ausência de efeitos do exercícios sob a percepção da dor ${ }^{(18,19)}$ e ainda há aqueles que apontam exacerbação da dor após o exercício físico ${ }^{(20)}$, sobretudo após 
exercícios de resistência ${ }^{(21,22)}$. Essas contradições podem ser devidas a características metodológicas das pesquisas. Por exemplo: a intensidade do exercício ao qual foram submetidos os participantes ${ }^{(23,24)}$, o tipo de exercício executado ${ }^{(25)}$, os diferentes protocolos de avaliação da dor ${ }^{(26,27)}$ ou treinamento ${ }^{(23-25)}$. Protocolos que avaliam elevadas intensidades de dor (como os protocolos de dor experimental isquêmica) reduzem a sensibilidade da medida álgica, reduzindo a probabilidade de identificar a ação inibitória proporcionada pelo exercício(26,27). Protocolos de treinamento realizados com intensidades muito baixas não atingem os efeitos fisiológicos proporcionados pelo exercício, reduzindo a probabilidade de detectar os efeitos sobre a dor ${ }^{(23,25)}$.

Embora contraditório, aceita-se que a atividade física seja benéfica no tratamento da dor. Esse fenômeno - analgesia induzida pelo exercício - é sobretudo confirmado pelo aumento do limiar da dor em atletas quando comparados com a população não-atleta ${ }^{(28,29)}$. Contudo, os efeitos neurofisiológicos que explicam esse fenômeno ainda são incertos. Uma das hipóteses mais aceitas é a influência do exercício nos mecanismos endógenos de controle da dor ${ }^{(28)}$.

A compreensão dos efeitos do exercício sobre a dor crônica complexifica-se pelas características clínicas da própria manifestação clínica das síndromes dolorosas. Tanto causa quanto neurofisiologia da dor crônica ainda são mecanismos estudados e discutidos pela literatura científica. Neste artigo apresentamos e discutimos hipóteses neurofisiológicas da analgesia induzida pelo exercício. A primeira parte descreve os mecanismos endógenos de modulação da dor. Em seguida, discutem-se os mecanismos envolvidos na analgesia induzida pelo exercício. Concluindo com considerações clínicas que favorecem efeitos neurofisiológicos benéficos do exercício em pacientes com dor crônica.

\section{MECANISMOS ENDÓGENOS DE CONTROLE DA DOR}

A dor é um fenômeno dinâmico. Ao longo de todo o trajeto nervoso, as aferências nociceptivas recebem inúmeras influências excitatórias e inibitórias de diferentes mecanismos de modulação da dor. Essa modulação do sinal nociceptivo (dor potencial) ocorre no sistema nervoso periférico (SNP) pela ação de neuromediadores (ex.: bradicinina, prostaglandinas e serotonina) e no sistema nervoso central (SNC) pela liberação de neurotransmissores (ex:: noradrenalina, serotonina, encefalinas e dopamina) ${ }^{(30)}$. O estímulo nociceptivo aciona receptores que, por sua vez, ativam fibras aferentes do SNP ( figura 1, região 1) que transmitem a mensagem de "lesão potencial" ao SNC (figura 1, regiões 2, 3 e 4). Essa comunicação entre do meio exógeno e endógeno (SNP e SNC) ocorre no corno posterior da medula espinhal pela sinapse entre o primeiro e segundo neurônio (ou por intermédio de um interneurônio).

A dor aguda diretamente associada à lesão tissular - decorre de um processo inflamatório caracterizado pelo calor, edema, vermelhidão e dor na região atingida ${ }^{(30)}$. Em condição normal, durante o processo inflamatório que ocorre na região periférica (ilustrado pelo antebraço na figura 1), tanto os receptores quanto as fibras nociceptivas (fibras $A \delta$ e C) podem ser sensibilizadas. Em resposta a um estímulo repetitivo (provenientes do foco inflamatório, por exemplo), ambas as estruturas podem manifestar redução do limiar de ativação. Esse fenômeno explica a sensibilização do local lesado. Mais especificamente, esse fenômeno ocorre pela liberação prolongada de alguns mediadores químicos, como a bradicinina, a histamina, a serotonina e as prostaglandinas. Esses mediadores facilitam a abertura dos canais de cálcio $(\mathrm{Ca}++)$ que despolarizam as terminações nervosas livres e modificam a síntese proteica pró-nociceptiva (ex.: citocinas, interleucinas e as neurotropinas). Esse fenômeno fisiológico de sensibilização local é denominado hiperalgesia primária(1).

Entretanto, a sensibilização não se manifesta apenas no local lesado. Geralmente, a região em torno da lesão também se apresenta hipersensível. A sensibilização de regiões afastadas da lesão ocorre

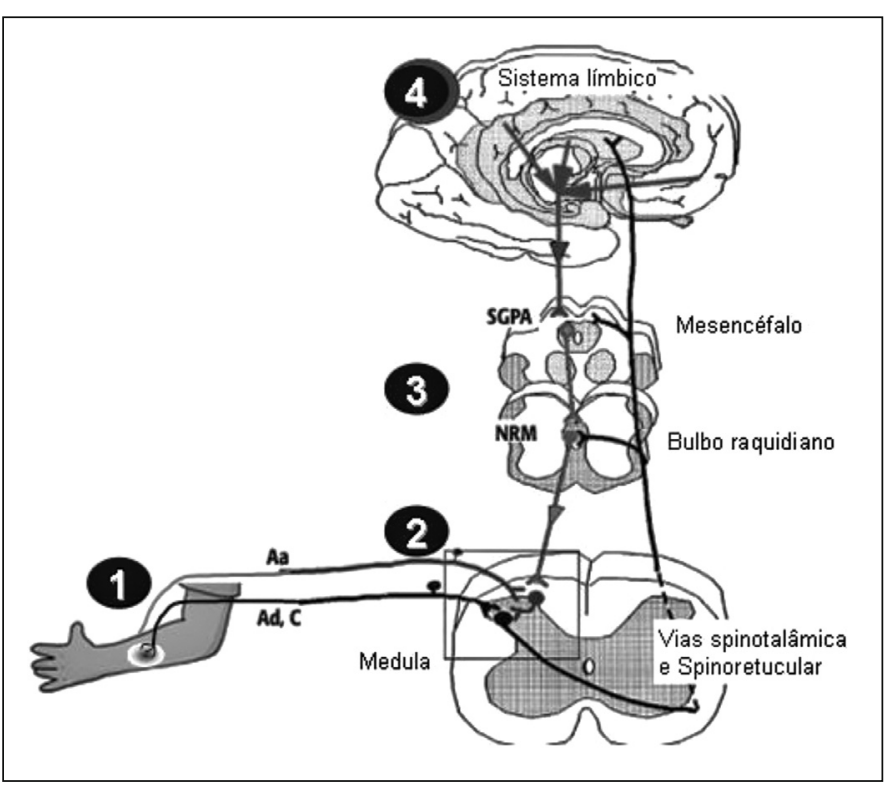

Figura 1. Níveis de modulação da dor. No nível 1 ilustra-se o sistema nervoso periférico, onde há a liberação de mediadores químicos que favorecem a estimulação das terminações nervosas. As informações dolorosas são transmitidas ao sistema nervoso central pelas fibras Ad e C. No nível 2 estamos no corno posterior da medula espinal. Nesse estágio ilustra-se a teoria do portão, descrita por Melzack e Wall (1968). Segundo essa teoria, estimulações das fibras Aa podem modular as aferências nociceptivas das fibras Ad e C. No nível 3, faz-se referência ao tronco cerebral, que inclui a substância cinza periaqueducal (SCPA), o núcleo de Raphe-Magnus (NRM) e o locus coeruleus. Finalmente, no estágio 4, representamos os centros superiores de controle da dor, sistema límbico e os córtex somatossensórios primário e secundário.

em consequência de um fenômeno central, denominado hiperalgesia secundária ${ }^{(31)}$. Ao contrario da hiperalgesia primária de causa periférica, a hiperalgesia secundária exprime aumento da atividade do segundo neurônio (na medula espinhal, ino 2 da figura 1) em reação a um estímulo constante. Um estímulo intenso e frequente provoca uma sensibilização central pela somação temporal do estímulo no axônio (wind up). Nesse caso, há aumento na convergência do fluxo nervoso periférico, que estimula o neurônio antes que ele esteja suficientemente despolarizado. Além disso, o fluxo nervoso se propaga distalmente a outras terminações livres da mesma fibra nervosa, sensibilizando regiões afastadas da lesão. Esse último fenômeno denomina-se reflexo axônico. Os nociceptores são capazes de se autossensibilizar, diretamente ou indiretamente. Esses mecanismos manifestam-se pela liberação de substâncias neuroativas como os peptídios (sobretudo a substância P) ${ }^{(1)}$ e os aminoacidos excitatórios (NMDA e AMPA) $)^{(32)}$.

Algumas dores crônicas podem manifestar-se pelo aumento dos mecanismos excitatório endógenos de controle da dor, ou ainda pela perda dos sistemas inibitórios. Por exemplo, a persistência da sensibilização central mesmo após a parada do estímulo frequenteu, ou uma somação temporal exagerada, onde a hiperalgesia e mesmo a alodinia persistem após o retorno ao repouso (ausência do estímulo repetitivo). Essa hipersensibilidade dos mecanismos excitatórios da dor é observada em indivíduos com dores orofaciais crônicas ${ }^{(33)}$, como síndrome do cólon irritável $^{(34)}$ e na fibromialgia ${ }^{(35)}$. Na figura 2 apresentamos um modelo de alodinia proposto por Cervero e Laird (1996). De acordo com esse modelo, a excitação dos nociceptores produzida pela lesão (periférica) atiça os interneurônios espinhais que mediam a despolarização aferente primária entre os mecanorreceptores de baixo limiar e os nociceptores. Dessa forma, estímulos com intensidade inferior à do limiar de dor podem ativar mecanorreceptores de baixa intensidade no local lesado e ser interpretados como nocivos, em certas circunstâncias (alodinia).

Ainda na medula espinhal, a interação entre as fibras aferentes nociceptivas (fibras $A \boldsymbol{\delta}$ e C) e as fibras não-nociceptivas (fibras A $\alpha$ e A $\beta$ )) 


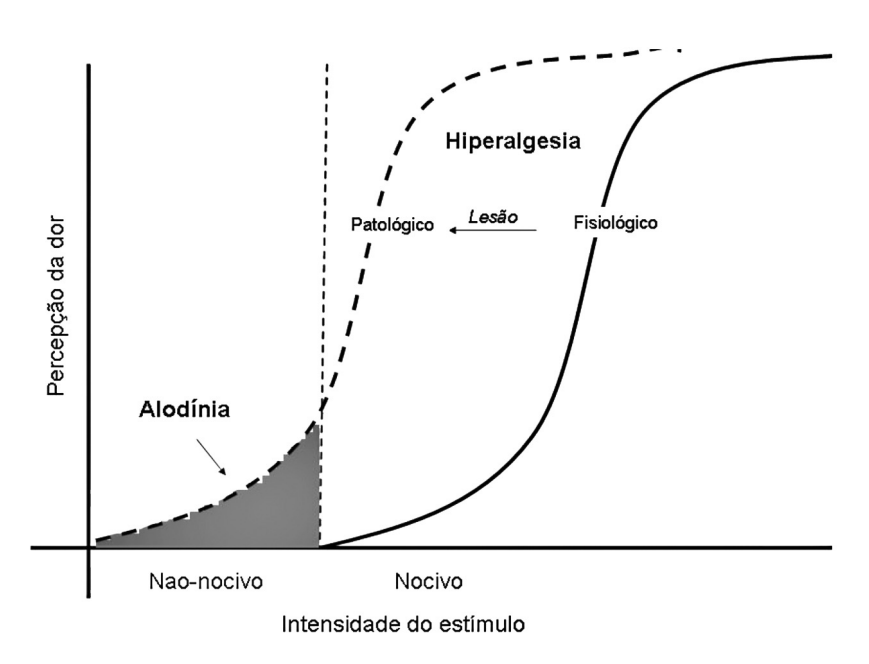

Figura 2. Modelo explicativo da alodinia e hiperalgesia (Cervero e Laird, 1996). Estímulos não nocivos podem ser interpretados como nocivos em condições patológicas de lesão periférica ou por plasticidade do sistema nervoso central.

podem desencadear uma analgesia localizada. Por exemplo, uma massagem leve na região lesada ativa as fibras não-nociceptivas que, por sua vez, ativam um interneurônio que inibe a transmissão do impulso nervoso das fibras nociceptivas. Esse fenômeno ocorre no corno posterior da medula e é conhecido sob o nome de "teoria do portão"(37). Contudo, a hiperatividade do interneurônio na medula espinhal também pode produzir sensibilização central (como visto anteriormente para o segundo neurônio) (figura 3). Esse aumento na excitabilidade é ativado pelos receptores NMDA na medula espinhal e se manifesta clinicamente pela alodinia, ou seja, um estímulo não-nociceptivo é interpretado como dor ${ }^{(38)}$. Essa situação pode ser ilustrada pela dor ressentida após uma queimadura por exposição ao sol, onde um simples toque na pele produz uma sensação dolorosa. Nesse caso, o impulso nervoso das fibras não-nociceptivas é interpretado como dor.

O segundo neurônio fará conexões com o córtex por intermédio do tálamo. Porém, antes de atingir os centros superiores, esse neurônio faz conexões com o tronco cerebral (núcleo rafe magno e substância cinzenta periaquedutal), onde ativa os mecanismos de controle inibitório

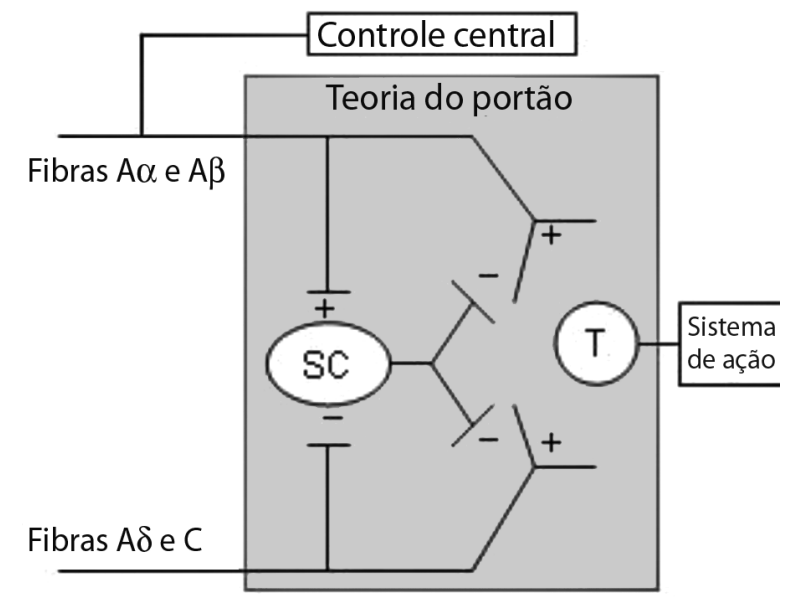

Figura 3. Esquema representativo da teoria do portão modificada. Nesse esquema representativo temos as fibras não-nociceptivas ( $\mathrm{Aa}$ e $\mathrm{Ab}$ ) ativando um interneurônio (SG), que inibe as fibras nociceptivas (Ad e C), estando todo esse mecanismo subordinado ao córtex cerebral (controle central). A teoria do portão modificada considera que esse mecanismo está subordinado aos aspectos cognitivos, afetivos e emocionais (Melzack, 1999). difuso nociceptivo (CIDN) ${ }^{(39)}$ (figura 1, região 3). Esse mecanismo descendente de inibição da dor é ativado por um estímulo nociceptivo. Ao ser ativado, fibras noradrenérgicas e serotoninérgicas favorecem a liberação de encefalinas nos cornos posteriores da medula espinhal. Essa liberação de encefalinas inibe a percepção da dor de forma difusa ${ }^{(40-42)}$.

Déficit nos mecanismos endógenos inibitórios de controle da dor, ou seja os CIDN, tem sido apontado como possível causa de alguns tipos de dor crônica ${ }^{(43,44)}$. Pesquisas recentes confirmam a hiperalgesia de pacientes com fibromialgia e apontam o déficit na ação do CIDN. Através do protocolo de somação espacial da dor ${ }^{(45)}$, Julien et al. ${ }^{(46)}$ observam que, ao contrário dos sujeito sadios e de pacientes com lombalgias que demonstram redução da dor após imersão de todo o membro superior em água quente ${ }^{(46)}$ ou fria(47), nos fibromiálgicos há não há redução significativa da dor, havendo em alguns casos exacerbação desta. Essa ausência de ação dos mecanismos endógenos de controle da dor também tem sido observada em pacientes com cefaleias do tipo tensional ${ }^{(44)}$. Acredita-se que esse déficit em pacientes com dores crônicas seja promovido pela baixa concentração de serotonina e noradrenalina no SNC.

Nos centros superiores, o estímulo nociceptivo é enfim interpretado como dor (figura 1, região 4), onde sua componente sensório-discriminante (localização e intensidade da dor) é interpretada na região somatossensorial primária e secundária. A componente afetiva da dor é interpretada em diversas estruturas límbicas, sobretudo no córtex insular e córtex cingular anterior ${ }^{(48)}$. Nesse estágio, a percepção da dor é influenciada pelas experiências anteriores, pelas crenças e pelo contexto em que o indivíduo se encontra no momento da lesão. Estudos recentes demonstram que as expectativas de efeito nocebo e placebo influenciam a modulação da dor, podendo tanto inibir quanto facilitar a ação dos mecanismos endógenos de controle da dor, como a teoria do portão (figura 3) $)^{(49)}$ e o CIDN (figura 1) $)^{(50)}$.

Dentre outras alterações fisiológicas e neurofisiológicas observadas no contexto de dores persistentes, há alterações na resposta ao estresse, desequilibro do SNA e a hiperalgesia por uso contínuo de opioides. Resumidamente, os mecanismos de resposta ao estresse se caracterizam pela perda do feedback inibitório da hipófise, inibindo a liberação de cortisol e adrenalina ${ }^{(51,52)}$. O desequilíbrio do SNA se manifesta sobretudo pela predominância da atividade simpática nos indivíduos com dores crônicas ${ }^{(53,54)}$. O uso de farmacológicas analgésicos, como os opioides, também pode induzir adaptações pró-nociceptivas ${ }^{(55)}$. Embora sejam excelentes analgésicos nos pacientes com dor crônica(56) o uso a longo prazo pode também induzir hiperalgesia, que parece ser dependente dos receptores NMDA, pois a administração simultânea de antagonistas ao NMDA (ex.: Ketamina) parece prevenir esse efeito.

\section{ANALGESIA INDUZIDA PELO EXERCÍCIO}

O fenômeno da analgesia induzida pelo exercício (AIE) é sobretudo observado em atletas. Os primeiros relatos descrevem bailarinas que continuavam a atividade mesmo havendo sofrido lesões graves e afirmavam não terem ressentido dor durante a execução dos movimentos. Essa característica se manifesta sobretudo no contexto competitivo. 0 primeiro a demonstrar esse fenômeno foi o médico Black, em 1979; em seu experimento, houve aumento no limiar de dor imediatamente após uma corrida de 40 minutos. Quase 30 anos após esse primeiro estudo, os mecanismos envolvidos ainda são desconhecidos, embora haja tendência a aceitar a ação do sistema endógeno opioide(28).

\section{POPULAÇÃO SAUDÁVEL E ATLETAS}

Durante um evento importante na carreira, o atleta pode se lesar durante a execução de uma atividade, sem, no entanto, perceber a dor. Havendo, nesse caso, aumento nos limiares de percepção e tolerância da dor. Embora casos esportivos de lesão ocorrida durante 
a competição com percepção tardia da dor possam ser citados, a justificativa neurofisiológica dessa inibição nas vias de dor permanece desconhecida. Um dos mecanismos indiretamente envolvidos, no contexto competitivo, é a analgesia induzida pelo estresse, pela ação do eixo hipotálamo-hipofisário liberando cortisol e adrenalina(52,57-59) , ou, ainda pela componente psicológica no controle da dor ${ }^{(48,50,60)}$. Contudo a analgesia induzida pelo exercício também é relatada em outras situações, como, por exemplo, na ausência de qualquer estresse aparente, ao repouso; observa-se que atletas têm limiar à dor experimental significativamente e clinicamente superior ao de não atletas ${ }^{(61)}$.

Algumas hipóteses são propostas para explicar o aumento do limiar da dor em atletas ou em pessoas ativas comparados com os sedentários. Como mencionado acima, a hipótese neurofisiológica mais explorada e aceita em seres humanos é, sem dúvida, a analgesia induzida pela liberação de opioides ${ }^{(62)}$. Segundo Koltyn ${ }^{(28)}$, o estímulo constante das fibras descendentes neuromotoras favoreceria a liberação de opioides endógenos. aumentando assim o limiar de dor, pela redução da excitabilidade da membrana plasmática do neurônio, sobretudo das fibras aferentes do tipo A $\delta$. Dessa forma, quanto maior o nível de atividade do individuo, maior a liberação de $\beta$-endorfinas e met-encefalinas (peptídios opioides endógenos) ${ }^{(63)}$ que permitem a redução da percepção da dor. A maioria dos estudos que observou esse fenômeno AlE relata dependência do nível de atividade física, o qual teria que ser em intensidade extrema para ter a ação analgésica. Porém, recentemente, Bement e Sluka ${ }^{(64)}$ observaramm que atividade de intensidade moderada, como a marcha, induz a analgesia pela ação opioide em ratos. Estudo semelhante foi realizado com seres humanos, observando-se que a AIE depende da intensidade do exercício, devendo ser superior a 50\% do $\mathrm{VO}_{2 \max }$ (corrida) e a atividade deve durar mais que 10 minutos ${ }^{(65)}$. Outro fato demonstrado há alguns anos é a liberação de betaendorfinas no sangue e a regularidade da prática de exercício. Observa-se uma curva exponencial. Para uma mesma intensidade e duração de exercício, aqueles que praticam regularmente a atividade física liberam exponencialmaente mais endorfinas que sedentários ${ }^{(63)}$.

Embora, a ação dos opioides seja a mais aceita, sendo frequentemente observado o aumento da atividade opioide endógena com o exercício ${ }^{(66,67)}$ em nível sanguíneo ${ }^{(63,68)}$ como no liquor cefalorraquidiano ${ }^{(67)}$, com aumento de betaendorfinas no tálamo, hipotálamo ventral e substância cinza periaquiducal)(28). Alguns estudos ${ }^{(62,69)}$ observam que o fenômeno não depende (apenas) da ação dos opioides, pois há alteração nos limiares de dor de atletas mesmo com a administração de naloxona intravenosa (antagonista opioide). A duração média dessa analgesia induzida pela atividade aeróbica é de 15 a 30 minutos $^{(69,70)}$, podendo variar conforme as características de intensidade, duração e tipo de exercício.

Outros estudos observam o aumento das catecolaminas (dopamina e noradrenalina) durante o exercício(29,69). A dopamina vem sendo apresentada nos últimos anos como um neurotransmissor protetor da dor no SNC e está vinculada ao prazer e a motivação(71), porém, o aumento na concentração de dopamina após o exercício pode estar simplesmente associado ao estresse produzido pela atividade (Howells et al., 2005)(72). A noradrenalina também aumenta após o exercício ${ }^{(73)}$ e pode influenciar a AIE pela sua participação na modulação da dor, entre outras. na ativação das vias descentes de inibição da dor ${ }^{(74)}$, da mesma forma que o aumento na concentração de serotonina após o exercício ${ }^{(73)}$. O hormônio de crescimento, cuja secreção está diretamente dependente da carga e da frequência do exercício, também participa da modulação da dor e pode induzir a AlE ${ }^{(75)}$. A carência de sono, por sua vez, reduz a concentração de secreção do hormônio e pode causar dores difusas similares às percebidas por indivíduos com fibromialgia(76). A ação gaba, cuja secreção espinhal também é influenciada pelo exercício ${ }^{(77)}$, parece agir no processo da AlE, reduzindo a intensidade da dor pela liberação de gaba no SNC.
O SNA também parece influenciar a modulação da dor, com sua ação simpática (opioide) e a parassimpática (não-opioide e dopamínica) ${ }^{(78)}$. Durante o sintoma doloroso, observa-se predominância do SNA simpático (SNS), sobretudo nos homens ${ }^{(53)}$, havendo desequilíbrio do SNA pela predominância do SNS. Há evidência dos opioides como inibidores parciais da atividade do SNS, assim como inibição do SNS pode ser observada após o exercício físico ${ }^{(79)}$. Dessa forma, acredita-se que o exercício possa auxiliar no reequilíbrio do SNA e no controle da dor.

Segundo a teoria da rede neural(49) a interpretação da dor está longe de ser compreendida dentro de um contexto linear. A interação entre os diferentes neurotransmissores, as vias inibitórias e excitatórias da dor, as características motivacionais, psicológicas e contextuais influenciam continuamente a interpretação e percepção do sintoma álgico; além disso, uns influenciam os outros, formando uma complexa rede de comunicação. Por exemplo, os peptídios opioides interagem com a liberação hormonal da glândula pituitária e das catecolaminas. Esses neurotransmissores, dopamina, serotonina e noradrenalina, participam na modulação da dor; suas concentrações e proporções entre eles podem favorecer a analgesia, assim como a algesia(74). A modulação da dor não é determinada por um simples mecanismo ou neurotransmissor, mas pela interação de vias e interneurônios no SNC(74).

Cabe lembrar que o exercício também induz, paradoxalmente, hiperalgesia. Esse outro fenômeno é descrito tanto em atletas ${ }^{(80)}$ quanto em pacientes com dores ${ }^{(81)}$. Esse fenômeno, ao contrário da AlE, foi mais estudado e é melhor compreendido. A manutenção de uma contração muscular (seja durante o exercício ou por um mecanismo de proteção autônomo, compensatório para a "inibição" da dor pela inibição do movimento) aumenta a pressão intramuscular (pela atividade continua das fibras musculares, há redução no aporte sanguíneo (hemoglobinas e logo redução do aporte em oxigênio nesta região). Com a redução do aporte de $\mathrm{O}_{2}$, há predominância do metabolismo anaeróbio sobre o aeróbio, o que produz alterações químicas na região, como aumento das prostaglandinas, do glutamato, da serotonina e do piruvato e acido lático na periferia ${ }^{\left({ }^{81}\right)}$. O aumento na concentração das três primeiras está correlacionado com a intensidade de dor percebida. Dessa forma, há redução no limiar de ativação dos nociceptores ${ }^{(81,82)}$. Porém, o paradoxo da hiperalgesia induzida pelo exercício (HIE) é um fenômeno que ocorre somente no SNP e não no $\mathrm{SNCl}$, onde o mecanismo é relativamente menos complexo e mais compreendido. Com o repouso e retorno do fluxo normal da circulação, HIE é aliviada ${ }^{(80)}$.

\section{POPULAÇÃO COM DOR CRÔNICA}

A dor crônica, ao contrario da dor aguda, consiste em uma patologia em si, sendo reconhecida como tal pela 10a Revisão do Código Internacional das Doenças - Organização Mundial de Saúde. Estima-se que 19\% da população brasileira sofram de dores crônicas devido a doenças reumáticas, com predominância em mulheres, adultos jovens (idade média 37 + 27 anos), desempregados e com nível socioeconômico baixo (classe D) ${ }^{(83)}$. Além das dores crônicas de origem reumática, há também aquelas de origem musculoesquelética, como a lombalgia crônica e cefaleias do tipo tensional; viscerais, como a síndrome do cólon irritável; e neuropáticas, como a dor fantasma (relato de dor no membro amputado).

Seu tratamento é complexo, sendo propostos programas multidisciplinares para combater a plasticidade do SNC formada pela persistência do sintoma álgico. Dentre as características dos programas multidisciplinares, predomina a prescrição de exercícios aeróbicos, de fortalecimento e alongamentos ${ }^{(84,85)}$. Dessa forma, a literatura sobre a dor e a atividade física apresenta-se mais diversificada, observando o efeito dos diferentes tipos de exercícios nessa população.

A atividade física, seja ela cardiovascular, de fortalecimento ou alongamento, está associada ao bem-estar físico, mental e à inclusão social 
dos indivíduos. Estudos clínicos demonstram que a atividade física reduz a intensidade da dor relatada por pacientes com dores osteoarticulares ${ }^{(17)}$, lombares ${ }^{(86,87)}$, fibromiálgicas ${ }^{(15,88)}$, cervicais ${ }^{(82)}$, entre outras. Embora haja destaque aos benefícios clínicos do exercício reduzindo a intensidade da dor crônica, os efeitos fisiológicos envolvidos ainda são incertos; algumas vezes, o efeito analgésico é contraditório.

Estudos recentes associam a redução da dor à prática da atividade cardiovascular ${ }^{(19 ; 21)}$; a intensidade da atividade demonstra-se fundamental para o sucesso do tratamento. Comparando o efeito da atividade física de alta e baixa intensidade em pacientes com fibromialgia, van Santen et al.(24) observam aumento significativo no bem-estar global dos pacientes que realizaram 20 semanas de atividade física de alta intensidade e nenhuma melhora nos que praticaram atividade física de baixa intensidade no mesmo período. Contudo, os pacientes que praticaram exercício de alta intensidade também apresentaram aumento na intensidade da dor (9 milímetros na escala visual analógica). A exacerbação da dor, com exercícios de alta intensidade, é confirmada por outros autores ${ }^{(22,89)}$. Para atingir o efeito analgésico, estudos clínicocientíficos $^{(15,82,90)}$ destacam a importância da adaptação dos exercícios às condições físicas e fisiológicas dos pacientes ${ }^{(91)}$, que, além de favorecer a atividade física sem a exacerbação do sintoma álgico, tendem a reduzir a taxa de abandono da atividade.

Com um programa multidisciplinar que incluía oito semanas de caminhada com uma frequência de três vezes semanais, duração de 20 a 30 minutos, com intensidade de 40-60\% frequência cardíaca máxima), demonstramos redução de $26 \%$ da dor clínica e aumento de $34 \%$ no limiar da dor experimental de pacientes com fibromialgia(85).

O efeito do alongamento é sobretudo fisiológico, onde o aumento do comprimento do músculo ao repouso e o relaxamento proporcionados pelo exercício favorecem o fluxo sanguíneo e reduzem a hiperalgesia induzida pela contração muscular (tensão), como a HIE descrita acima. Valim et al. ${ }^{(90)}$ relatam que o alongamento melhora a saúde mental, além de reduzir a dor clínica dos indivíduos com fibromialgia. Além disso, o alongamento e o fortalecimento da musculatura também têm um efeito mecânico; eles auxiliam no reequilíbrio musculoesquelético, causa parcial de dores crônicas como as lombalgias, cervicalgias e mesmo fibromialgias.

Finalmente, o exercício aeróbio é o mais descrito e estudado na população sadia e atlética e muito utilizado no tratamento da dor crônica. Há muitos relatos de redução na percepção da dor após um período de treinamento, em indivíduos com dor crônica ${ }^{(15,17)}$. Os efeitos dos exercícios também são visíveis na lombalgia crônica ${ }^{(86)}$. Embora haja evidências clínicas do efeito do exercício no controle da dor crônica, as alterações nos mecanismos endógenos de modulação da dor podem justificar diferenças no efeito agudo do exercício. Alguns estudos(22,89) relatam aumento importante na dor de indivíduos com fibromialgia durante e após a contração muscular. Além de estudos mais aprofundados para compreender os efeitos neurofisiológicos do exercício na dor cronica, esses relatos devem ser considerados na clínica, onde o exercício terá como efeito agudo hiperalgesia significativamente maior que em outras populações. Na figura 4 representamos de forma esquemática como o exercício pode agir em cada nível dos mecanismos endógenos de controle da dor.

\section{LIMITES DOS ESTUDOS}

Os principais limites dos estudos de AlE em indivíduos saudáveis e atléticos são: (a) pequenas amostras (10 a 15 indivíduos); (b) amostras com predominância masculina, mulheres são raramente representadas; (c) variação nas doses de naloxona (variando de 0,8 a 30mg) pode explicar a alteração nos resultados entre estudos, pois sabe-se que a concentração pode proporcionar um efeito antagonista ou agonista do sistema opioide; (d) a maioria dos estudos observa o limiar de tolerância

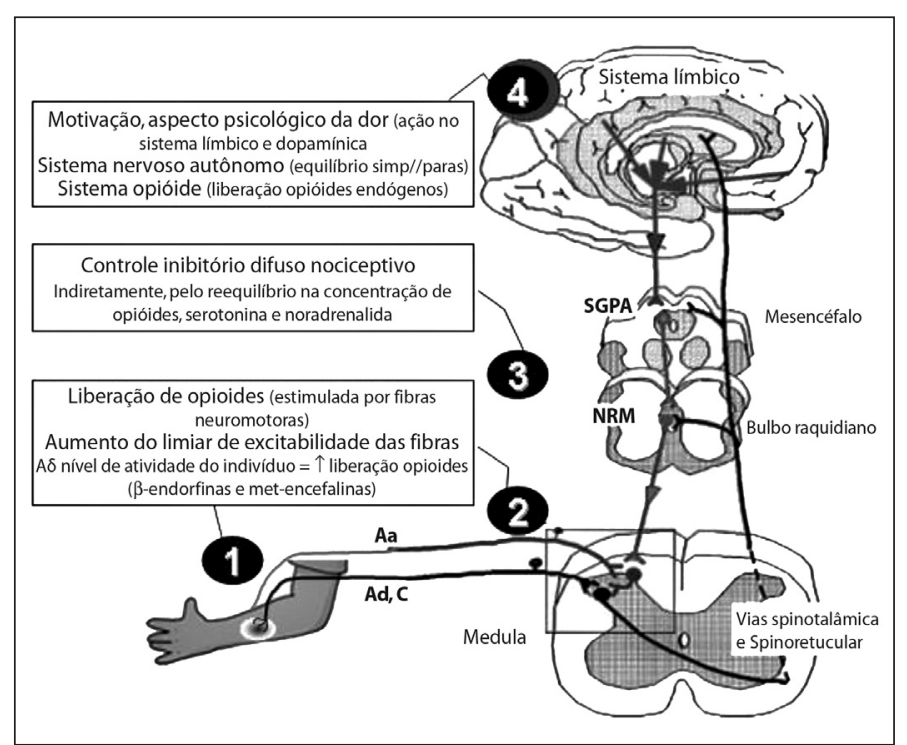

Figura 4. Hipótese da ação do exercício nos mecanismos endógenos de controle da dor. No nível 1, sistema nervoso periférico; nível 2, corno posterior da medula espinal; nível 3, tronco cerebral; e nível 4, centros superiores de controle da dor.

e de intensidade da dor e o sistema opioide (por intermédio da naloxona). Nenhum estudo observa a ação dos mecanismos endógenos de controle da dor; (e) poucos estudos se interessam pelos efeitos agudos e crônicos do exercício nos indivíduos saudáveis e com dor; e (f) há uma lacuna na avaliação dos efeitos de exercícios de fortalecimento e alongamentos sobre a dor de indivíduos sadios e atléticos; porém, dadas as características dos mecanismos endógenos de controle da dor, acredita-se que nessa população os efeitos neurofisiológicos proporcionados por esses exercícios sejam discretos. Dentre os principais limites dos estudos dos efeitos neurofisiológicos do exercício sobre a dor, destacase, sobretudo, a raridade dos que utilizaram dor experimental em seus protocolos; a variável dor é geralmente avaliada de forma clínica.

\section{CONCLUSÃO}

O exercício físico, sobretudo o aeróbico, interage como modulador do aspecto desagradável da dor por intermédio do córtex, motivacional psicológico e da dopamina; no SNA (dopamina e opioides); nos mecanismos descendentes (noradrenalina, serotonina e peptídios opioides); na medula espinhal, (opioide, gaba, fibras A $\delta$ ). Ao contrário do que era proposto na década de 1990, o exercício aeróbico não precisa ser de alta intensidade ou de intensidade submáxima para ter um efeito sobre a dor. Estudos realizados nos últimos cinco anos demonstram que o exercício físico aeróbico de intensidade moderada, mantido por mais de 10 minutos, pode ativar os mecanismos endógenos de controle da dor (em indivíduos sadios).

Em suma, indivíduos com dores crônicas devem passar por avaliação sensitiva, para verificar a presença de distúrbios na modulação da dor, como a anodinia e hiperalgesia; e avaliação biomecânica funcional, para verificar a presença de desequilíbrios musculares e instabilidade articular que possam causar dor crônica de origem mecânica. Os exercícios de alongamento e fortalecimento são prescritos em função das observações clínicas, mas, para favorecer a adesão ao tratamento, metas pessoais devem ser previamente negociadas com os indivíduos. A atividade cardiovascular é essencial para o reequilíbrio neuro-hormonal, podendo ser de intensidade moderada (40 a 60\% FC $C_{\text {max }}$ ), no mínimo durante 10 minutos. Para favorecer os efeitos fisiológicos e neurofisiológicos, sugere-se que essa atividade seja em uma frequência trissemanal.

Todos os autores declararam não haver qualquer potencial conflito de interesses referente a este artigo. 


\section{REFERÊNCIAS BIBLIOGRÁFICAS}

1. Le Bars D, Willer JC. Physiologie de la douleur. EMC-Anesthésie Réanimation. 2004;(1):227-66.

2. IASP - Classification of chronic pain. Pain. 1986;Suppl 3:S1-\$226.

3. Elliott AM, Smith BH, Penny KI, Smith WC, Chambers WA. The epidemiology of chronic pain in the community. Lancet. 1999;354(9186):1248-52.

4. Gureje O, Von KM, Simon GE, Gater R. Persistent pain and well-being: a World Health Organization Study in Primary Care. JAMA. 1998;280(2):147-51.

5. Kerns R, Otis J, Rosenburg R, Reid C. Veterans'reports of pain and associations with ratings of health, healthrisk behaviors, affective distress, and use of the healthcare system. J Rehabil Res Dev. 2003;40(5):371-80.

6. Holtz WV, Stechman Neto J. Epidemiologia da dor em pacientes de Curitiba e região metropolitana. Revista Dor. 2008;9(8):1217-24

7. Lawrence RC, Felson DT, Helmick CG, Arnold LM, Choi H, Deyo RA, et al. Estimates of the prevalence of arthritis and other rheumatic conditions in the United States. Part Il. Arthritis \& Rheumatism. 2008:58(1):26-35.

8. Zeller JL, Burke AE, Glass RM. JAMA patient page. Acute pain treatment. JAMA. 2008;299(1):128

9. Shang $A B$, Gan TJ. Optimising postoperative pain management in the ambulatory patient. Drugs. 2003:63(9):855-67.

10. Turk DC, Dworkin RH, Burke LB, Gershon R, Rothman M, Scott J, et al. Developing patient-reported outcome measures for pain clinical trials: IMMPACT recommendations. Pain. 2006:125(3):208-15.

11. Faas A. Exercises: which ones are worth trying, for which patients, and when? Spine. 1996;21 (24):2874-8.

12. Sewitch MJ, Dobkin PL, Bernatsky S, Baron M, Starr M, Cohen M, et al. Medication non-adherence in women with fibromyalgia. Rheumatology (Oxford). 2004;43(5):648-54.

13. Flor H, Hermann C. Biopsychosocial Models of Pain. In: Dworkin RH, Breitbart WS, editors. Psychosocial Aspect of Pain: A Handbook fot Health Care Providers. Seattle: IASP Press; 2004. 47-75.

14. Bennett RM, Burckhardt CS, Clark SR, O'Reilly CA, Wiens AN, Campbell SM. Group treatment of fibromyalgia: A 6 month outpatient program. J Rheumatol. 1996;23(3):521-8.

15. Barcellos de Souza J, Charest J, Marchand S. École interactionnelle de fibromyalgie : description et évaluation. Douleur et Analgésie. 2007;20:213-8.

16. McCain GA, Bell DA, Mai FM, Halliday PD. A controlled study of the effects of a supervised cardiovascular fitness training program on the manifestations of primary fibromyalgia. Arthritis Rheum. 1988;31(9):1135-41

17. Lee HY, Lee KJ. [Effects of Tai Chi exercise in elderly with knee osteoarthritis]. Taehan Kanho. Hakhoe. Chi. $2008 ; 38(1): 11-8$. Ref Type: Abstract

18. Martin L, Nutting A, Maclntosh BR, Edworthy SM, Butterwick D, Cook J. An exercise program in the treatment of fibromyalgia. J Rheumatol. 1996;23(6):1050-3.

19. Sabbag LMS, Pastore CA, Yazbek Jr P, Miyazaki MH, Gonçalves A, Kaziyama HHS et al. Efeitos do condicionamento físico sobre pacientes com fibromialgia. Rev Bras Med Esporte. 2007;13(1):6-10.

20. Padawer WJ, Levine FM. Exercise-induced analgesia: fact or artifact? Pain. 1992;48(2):131-5.

21. Busch AJ, Schachter CL, Overend TJ, Peloso PM, Barber KA. Exercise for fibromyalgia: a systematic review. J Rheumatol. 2008 ;35(6):1130-44. Review.

22. Vierck CJ Jr, Staud R, Price DD, Cannon RL, Mauderli AP, Martin AD. The effect of maximal exercise on temporal summation of second pain (windup) in patients with fibromyalgia syndrome. J Pain. 2001;2(6):334-44

23. Schachter CL, Busch AJ, Peloso PM, Sheppard MS. Effects of short versus long bouts of aerobic exercise in sedentary women with fibromyalgia: a randomized controlled trial. Phys Ther. 2003;83(4):340-58.

24. van Santen M, Bolwijn P, Landewe R, Verstappen F, Bakker C, Hidding A, et al. High or low intensity aerobic fitness training in fibromyalgia: Does it matter? J Rheumatol. 2002;29(3):582-7.

25. Rooks DS, Silverman CB, Kantrowitz FG. The effects of progressive strength training and aerobic exercise on muscle strength and cardiovascular fitness in women with fibromyalgia: A pilot study. Arthritis Rheum. 2002;47(1):22-8.

26. Droste C, Greenlee MW. Comments on Padawer and Levine, PAIN, 48 (1992) 132-135. Pain. 1992;50(2):241-3.

27. Pertovaara A, Kemppainen P. Comments on Padawer and Levine, PAIN, 48 (1992) 132-135. Pain. 1992;50(2):239-40.

28. Koltyn KF. Analgesia following exercise: a review. Sports Med. 2000;29(2):85-98.

29. Thoren P, Floras JS, Hoffmann P, Seals DR. Endorphins and exercise: physiological mechanisms and clinical implications. Med Sci Sports Exerc. 1990;22(4):417-28.

30. Cousins M, Power I. Acute and postoperative pain. In: Wall PD, Melzack R, editors. Textbook of pain. 4 ed. Toronto: Churchill Livingstone, $2003 ; 447-91$.

31. Snijdelaar DG, Dirksen R, Slappendel R, Crul BJ. Substance P. Eur J Pain. 2000;4(2):121-35.

32. Carlton SM. Peripheral excitatory amino acids. Curr Opin Pharmacol. 2001;1 (1):52-6.

33. Merrill RL. Orofacial pain mechanisms and their clinical application. Dent Clin North Am. 1997;41 (2):167-88

34. Verne GN, Price DD. Irritable bowel syndrome as a common precipitant of central sensitization. Curr Rheumatol Rep. 2002;4(4):322-8.

35. Staud R, Vierck CJ, Cannon RL, Mauderli AP, Price DD. Abnormal sensitization and temporal summation of second pain (wind-up) in patients with fibromyalgia syndrome. Pain. 2001;91(1-2):165-75.

36. Cervero F, Laird JMA. Review article: Mechanisms of touch-evoked pain (allodynia): a new model. Pain 1996;68:13-23

37. Melzack R, Wall PD. Pain mechanisms: A new theory. Science 1965;150(699):971-9.

38. Marchand S. Le phénomène de la douleur. Chenelière/McGraw-Hill; 1998.

39. Bouhassira D, Villanueva L, Le BD. Effects of systemic morphine on diffuse noxious inhibitory controls: role of the periaqueductal grey. Eur J Pharmacol. 1992;216(2):149-56.

40. Le Bars D, Dickenson AH, Besson JM. Diffuse noxious inhibitory controls (DNIC). I. Effects on dorsal horn convergent neurones in the rat. Pain. 1979;6(3):283-304

41. Le Bars D, Dickenson AH, Besson JM. Diffuse noxious inhibitory controls (DINC). II. Lack of effect on nonconvergent neurones, supraspinal involvement and theorical implications. Pain. 1979:6(3):305-27.

42. Willer JC, Bouhassira D, Le Bars D. [Neurophysiological bases of the counterirritation phenomenon: diffuse control inhibitors induced by nociceptive stimulation]. Neurophysiol Clin. 1999;29(5):379-400

43. Julien N, Souza JB, Charest J, Marchand S. Déficits des mécanismes endogènes de contrôle de la douleur dans la fibromyalgie. Congrès de l'Association des Fibromyalgiques 2006.

44. Pielsticker A, Haag G, Zaudig M, Lautenbacher S. Impairment of pain inhibition in chronic tension-type headache. Pain. 2005;118(1-2):215-23.

45. Marchand S, Arsenault P. Spatial summation for pain perception: interaction of inhibitory and excitatory mechanisms. Pain. 2002;95(3):201-6

46. Julien N, Goffaux P, Arsenault P, Marchand S. Widespread pain in fibromyalgia is related to a deficit of endogenous pain inhibition. Pain. 2005;114(1-2):295-302

47. Souza JB, Potvin S, Goffaux P, Charest J, Marchand S. The deficit of pain inhibition in fibromyalgia is more pronounced in patients with comorbid depressive symptoms. Clin J Pain. In press 2008.

48. Rainville P, Duncan GH, Price DD, Carrier B, Bushnell MC. Pain affect encoded in human anterior cingulate but not somatosensory cortex. Science; 1997;277(5328):968-71.
49. Melzack R. From the gate to the neuromatrix. Pain. 1999;Suppl 6:S121-6.

50. Goffaux P, Redmond WJ, Rainville P, Marchand S. Descending analgesia - when the spine echoes what the brain expects. Pain. 2007;130(1-2):137-43.

51. Crofford L, Pillemer SR, Kalogeras KT, Cash JM, Michelson D, Kling MA, et al. Hypothalamic-pituitaryadrenal axis perturbations in patients with fibromyalgia. Arthritis Rheum. 1994;37(11):1583-92.

52. Crofford LJ. Neuroendocrine abnormalities in fibromyalgia and related disorders. Am J Med Sci. 1998;315(6):359-66.

53. Tousignant-Laflamme $Y$, Rainville $P$, Marchand S. Establishing a link between heart rate and pain in healthy subjects: a gender effect. J Pain. 2005;6(6):341-7.

54. Martinez-Lavin M, Hermosillo AG, Rosas M, Soto ME. Circadian studies of autonomic nervous balance in patients with fibromyalgia: a heart rate variability analysis. Arthritis Rheum. 1998;41(11):1966-71.

55. Simonnet G, Rivat C. Opioid-induced hyperalgesia: Abnormal or normal pain? Neuroreport. 2003;14(1):1-7.

56. Vallerand AH. The use of long-acting opioids in chronic pain management. Nurs Clin North Am. 2003;38(3):435-45.

57. Crofford $\amalg$. The hypothalamic-pituitary-adrenal axis in the pathogenesis of rheumatic diseases. Endocrinol Metab Clin North Am. 2002;31(1):1-13.

58. Crofford L, Young EA, Engleberg NC, Korszun A, Brucksch CB, McClure LA, et al. Basal circadian and pulsatile ACTH and cortisol secretion in patients with fibromyalgia and/or chronic fatigue syndrome. Brain Behav Immun. 2004;18(4):314-25

59. Crofford LJ. The relationship of fibromyalgia to neuropathic pain syndromes. J Rheumatol Suppl. 2005;75:41-5.

60. Rainville P, Carrier B, Hofbauer RK, Bushnell MC, Duncan GH. Dissociation of sensory and affective dimensions of pain using hypnotic modulation. Pain. 1999;82(2):159-71.

61. Guieu R, Blin O, Pouget J, Serratrice G. Nociceptive threshold and physical activity. Can J Neurol Sci. 1992;19(1):69-71.

62. Olausson H, Charron J, Marchand S, Villemure C, Strigo IA, Bushnell MC. Feelings of warmth correlate with neural activity in right anterior insular cortex. Neurosci Lett. 2005;389(1):1-5.

63. Bouix $\mathrm{O}$, Najimi $\mathrm{S}$, Orsetti A. Mise en jeu et rôles physiologiques des peptides opioides endogènes dans l'adaptation à l'exercise physique. Science \& Sports. 1997;12:26-40.

64. Bement MK, Sluka KA. Low-intensity exercise reverses chronic muscle pain in the rat in a naloxonedependent manner. Arch Phys Med Rehabil. 2005;86(9):1736-40.

65. Hoffman R, Al'Absi M. The effect of acute stress on subsequent neuropsychological test performance (2003). Arch Clin Neuropsychol. 2004;19(4):497-506.

66. Manning EL, Fillingim RB. The influence of athletic status and gender on experimental pain responses. J Pain. 2002;3(6):421-8

67. Hoffmann $P$, Terenius $L$, Thoren $P$. Cerebrospinal fluid immunoreactive beta-endorphin concentration is increased by voluntary exercise in the spontaneously hypertensive rat. Regul Pept. 1990:28(2):233-9.

68. Janal MN, Colt EW, Clark WC, Glusman M. Pain sensitivity, mood and plasma endocrine levels in man following long-distance running: Effects of naloxone. Pain. 1984;19(1):13-25.

69. Droste C, Greenlee MW, Schreck M, Roskamm H. Experimental pain thresholds and plasma beta-endorphin levels during exercise. Med Sci Sports Exerc. 1991;23(3):334-42.

70. Koltyn KF, Garvin AW, Gardiner RL, Nelson TF. Perception of pain following aerobic exercise. Med Sci Sports Exerc. 1996;28(11):1418-21.

71. Wood PB. A reconsideration of the relevance of systemic low-dose ketamine to the pathophysiology of fibromyalgia. J Pain. 2006;7(9):611-4.

72. Howells FM, Russell VA, Mabandla MV, Kellaway LA. Stress reduces the neuroprotective effect of exercise in a rat model for Parkinson's disease. Behav Brain Res. 2005:165(2):219-20.

73. Sarbadhikari SN, Saha AK. Moderate exercise and chronic stress produce counteractive effects on different areas of the brain by acting through various neurotransmitter receptor subtypes: a hypothesis. Theor Biol Med Model. 2006:3:33. Review.

74. Millan MJ. Descending control of pain. Prog Neurobiol. 2002;66(6):355-474.

75. Godfrey RJ, Madgwick Z, Whyte GP. The exercise-induced growth hormone response in athletes. Sports Med. 2003;33(8):599-613.

76. Moldofsky H, Scarisbrick P. Induction of neurasthenic musculoskeletal pain syndrome by selective sleep stage deprivation. Psychosom Med. 1976;38(1):35-44

77. Streeter CC, Jensen JE, Perlmutter RM, Cabral HJ, Tian H, Terhune DB, et al. Yoga Asana sessions increase brain GABA levels: a pilot study. J Altern Complement Med. 2007:13(4):419-26.

78. Cheshire WP Jr, Dickson DW, Nahm KF, Kaufmann HC, Benarroch EE. Dopamine beta-hydroxylase deficiency involves the central autonomic network. Acta Neuropathol. 2006;112(2):227-9.

79. Kemppainen P, Pertovaara A, Huopaniemi T, Johansson G, Karonen SL. Modification of dental pain and cutaneous thermal sensitivity by physical exercise in man. Brain Res. 1985:360(1-2):33-40.

80. Cook DB, O'connor PJ, Eubanks SA, Smith JC, Lee M. Naturally occurring muscle pain during exercise: assessment and experimental evidence. Med Sci Sports Exerc. 1997;29(8):999-1012.

81. Rosendal L, Kristiansen J, Gerdle B, Sogaard K, Peolsson M, Kjaer M, et al. Increased levels of interstitial potassium but normal levels of muscle IL-6 and LDH in patients with trapezius myalgia. Pain, 2005;1 19(1-3):201-9.

82. Andersen LL, Kjaer M, Sogaard K, Hansen L, Kryger Al, Sjogaard G. Effect of two contrasting types of physical exercise on chronic neck muscle pain. Arthritis Rheum. 2008;59(1):84-91.

83. Senna ER, De Barros AL, Silva EO, Costa IF, Pereira LV, Ciconelli RM, et al. Prevalence of rheumatic diseases in Brazil: A study using the COPCORD approach. J Rheumatol. 2004;31(3):594-7.

84. Lemstra M, Olszynski WP. The effectiveness of multidisciplinary rehabilitation in the treatment of fibromyalgia: A randomized controlled trial. Clin J Pain. 2005;21(2):166-74.

85. Souza JB, Bourgault P, Charest J, Marchand S. Long-term efficacy of the Interactional School of Fibromyalgia - a randomized controlled study. Arch Phys Med Rehabil. In press 2008.

86. Macedo LG, Latimer J, Maher CG, Hodges PW, Nicholas M, Tonkin L, et al. Motor control or graded activity exercises for chronic low back pain? A randomised controlled trial. BMC Musculoskelet Disord. 2008:9(1):65.

87. Charest J, Lavignolle B, Chenard J-R, Provencher M, Marchand S. École interactionnelle du dos. Rhumatologie. 1994;48(8):221-37.

88. Sim J, Adams N. Systematic review of randomized controlled trials of nonpharmacological interventions for fibromyalgia. Clin J Pain. 2002;18(5):324-36

89. Kosek $E_{t}$ Ekholm J, Hansson P. Modulation of pressure pain thresholds during and following isometric contraction in patients with fibromyalgia and in healthy controls. Pain. 1996:64(3):415-23.

90. Valim V, Oliveira L, Suda A, Silva L, de Assis M, Barros Neto T, et al. Aerobic fitness effects in fibromyalgia. J Rheumatol. 2003:30(5):1060-9.

91. Jones KD, Clark SR, Bennett RM. Prescribing exercise for people with fibromyalgia. AACN Clin Issues. 2002;13(2):277-93 